\title{
Carcinoma Primario Bilateral de Trompas de Falopio
}

\author{
INFORME DE UN CASO
}

Dres.: Germán Uriza Gutierrez* y Francisco Martín**

\section{INTRODUCCION}

La trompa de falopio es asiento de muy diversa patología benigna y maligna; sin embargo las neoplasias malignas son extremadamente raras y su diagnóstico generalmente se hace como hallazgo anatomo-clínico, (1), (2), (6), (10), (12), (13), (15), (22), (27), (28), (30).

El primer caso de la literatura mundial fue descrito por Reynaud quién lo presentó a la Sociedad de Patólogos de Manchester en 1847. (2), (6), (28). El segundo caso lo describe Rokitanski en $1861(2),(6),(28)$, y el primer caso publicado en la literatura médica lo realizó Orthman en 1888 (2), (6), (11), de un caso clínico intervenido por Martín en 1866, (2).

En la literatura mundial se conocían 300 casos en 1930; 449 en 1945 y 694 en 1960 reunidos por Sedlis; 780 en

\section{Clínica Palermo de Bogotá.}

* Ginecólogo-obstetra,. Profesor asistente Pontificia Universidad Javeriana, Bogota.

* Patólogo clínico Palermo, Bogotá. Jefe Departamento de Patología INC. Bogotá.
1965 reunidos por Jones y 829 en 1967 (2), a los que se suman 26 casos informados por Kinzet en 1975 (16), 20 casos de Wolf en 1976 (33), 31 casos de Kubista en $1977(17), 24$ casos de Barth en 1980 (4), 22 casos de Raju en 1981 (24), 15 casos de Tamimi en 1981 (19), 37 casos de Engeler en 1981 (8), y los últimos 16 casos informados en la literatura mundial por Herhey, recopilados en 8 hospitales del estado de Colorado (USA) y publicados en marzo de 1981 con los cuales se completan un total de 1100 casos, desde el primero publicado por Orthman en 1888 (11). (Tabla No. 1).

El tumor es casi siempre unilateral (23) y sólo en un $26 \%$ se encuentra bilateral (21) (26), según lo reporta Sedlis en la recopilación de 694 casos de carcinomas primarios de trompas publicados por él en 1960 (2). La rareza de este tumor ginecológico queda demostrada en nuestro medio, con la revisión de 112.849 piezas quirúrgicas durante 18 años en el hospital San Juan de Dios de Bogotá, que solo arrojó dos casos de adenocarcinoma de trompa de falopio publicados por Cantillo (6). 
Tabla No. 1

CASOS INFORMADOS EN LA LITERATURA MUNDIAL DE 1847 a 1981

\begin{tabular}{|l|c|c|c|}
\hline \multirow{2}{*}{ Autor } & \multirow{2}{*}{ Año } & $\begin{array}{c}\text { Recopilación } \\
\text { Hospitalaria }\end{array}$ & $\begin{array}{c}\text { Recopilación } \\
\text { Mundial }\end{array}$ \\
\cline { 3 - 4 } Reynaud & 1847 & Uno & - \\
Rokytansky & 1861 & Uno & - \\
Orthman & 1888 & Uno & - \\
- & 1930 & - & 300 \\
- & 1945 & - & 449 \\
Sedlis & 1960 & - & 694 \\
Jones & 1965 & - & 780 \\
- & 1967 & - & - \\
Cantillo & 1971 & Dos & - \\
Kinzel & 1975 & 26 & - \\
Wolf & 1976 & 20 & - \\
Kubista & 1977 & 31 & - \\
Barth & 1980 & 24 & - \\
Raju & 1981 & 22 & - \\
Tamimi & 1981 & 15 & - \\
Engeler & 1981 & 37 & - \\
Hershey & 1981 & 16 & - \\
\hline Informados hasta mayo de & 1981, & \\
según Hershey (11). & & - \\
\hline
\end{tabular}

\section{Frecuencia}

Es el tumor maligno ginecológico más raro Su frecuencia oscila entre el $0.100 \%$ y el $1.400 \%$ de todas las neoplasias ginecológicas malignas, con un promedio de $0.487 \%$. (Tabla No. 2 ).

\section{Edad}

Es un tumor que se encuentra con mayor frecuencia en mujeres pre y postmenopáusicas. En la literatura revisada la edad menor fue de 18 años (2) y la mayor de 82 (11), con un promedio de edad de 54.5 años. (Tabla No. 3 ).

\section{Paridad}

Un gran porcentaje de pacientes con este tumor son nulíparas, que en nuestra revisión constituyeron el $40 \%$ de los casos (2) (27). Algunos autores lo encuentran asociado frecuentemente a esterilidad (27).

\section{DIAGNOSTICO}

Por su extrema rareza, su crecimiento silencioso inicial y su sintomatología poco característica, su diagnóstico presuntivo es muy difícil, llegando a él en laparotomías exploradoras por otra patología como hallazgo anatomo-clínico (1), (2), (3), (10), (12), (13), (15), (22), (27), (28), (30), (32). En ocasiones se le ha encontrado al practicar una cesárea por indicación obstétrica (27), en laparotomía por embarazo extrauterino (2) o en minilaparatomía para esterilización post-parto (28).

Tabla No. 2

FRECUENCIA DEL ADENOCARCINOMA DE TROMPA SEGUN DIFERENTES AUTORES

\begin{tabular}{|l|l|}
\hline \multicolumn{1}{|c|}{ Autor } & Frecuencia \\
\hline Hanton y Col. & $1.400 \%$ \\
Ahumada y Col. & $0.700 \%$ \\
Benitez Soto L. & $0.620 \%$ \\
Anaspach y Hoffman & $0.138 \%$ \\
Stern y Hanly & $0.138 \%$ \\
Montazze & $0.180 \%$ \\
Wilson & $0.500 \%$ \\
Hayden y Potter & $1.000 \%$ \\
Shinfeld & $0.300 \%$ \\
Andrade & $0.190 \%$ \\
Green y Scully & $0.100 \%$ \\
Niklasson & $1.200 \%$ \\
\hline
\end{tabular}

\section{X: 0.538}

Su diagnóstico presuncional solo se logra en un número reducido de casos, para Anderson solo en el $8 \%$ (2) y en nuestra revisión bibliográfica solo en el $2.41 \%$ de los casos. (Tabla No. 4). 
Tabla No. 3

EDAD

\begin{tabular}{|l|r|r|}
\hline \multicolumn{1}{|c|}{ Autor } & No. & X años \\
\hline H'Opfel & 1 & 65.0 \\
Mendéz & 1 & 55.0 \\
Cantillo & 2 & 54.0 \\
Tamimi & 15 & 54.8 \\
Kadziora & 1 & 73.0 \\
Tjiptohardjo & 1 & 59.0 \\
Starr & 1 & 26.0 \\
Hershey & 16 & 55.4 \\
Galle & 1 & 75.0 \\
Anderson & 1 & 51.0 \\
Nilklasson & 1 & 69.0 \\
Shinfeld & 1 & 40.0 \\
Deppe & 2 & 50.0 \\
Andrade & 3 & 37.0 \\
Boury & 2 & 52.0 \\
Douglas & 2 & 56.0 \\
\hline
\end{tabular}

\section{$X: 54.1$}

La ayuda que pueda prestar la citología cervico vaginal de rutina es muy poca, similar a lo que ocurre con el adenocarcinoma de ovario. Un estudio de Fox presenta 3 casos con citología clase $V$ para adenocarcinoma, en los cuales el estudio endocervical $y$ endometrial fueron normales, la laparotomía reveló dos casos de adenocarcinoma primario de ovario y un caso de adenocarcinoma primario de trompa derecha. (9) $\mathrm{H}^{\prime}$ opfel reporta un caso en el cual la citología cervico vaginal de rutina identificó posible adenocarcinoma de trompa, que fue comprobado a la laparotomía (12). Estos dos estudios nos indican que en casos de citologías positivas para adenocarcinoma, en los cuales los estudios del endocervix y el endometrio sean normales, la laparotomía es obligante.
Frecuentemente se encuentra asociado a otras afecciones de los anexos tales como salpingitis crónica o quistes ováricos (2), (6), (11), (21). También se ha encontrado asociado a leiomiomatosis (2), (10), (11), embarazo tubárico (2), endometriosis o adenomiosis (11), adenocarcinoma de ovario (11), adenocarcinoma endometrial (11), carcinoma in situ del cervix (19), fibrosis retroperitoneal (1) o salpingitis tuberculosa (31); afecciones que en muchos casos permiten el diagnóstico precoz del adenocarcinoma de trompa.

Se destacan como medios que facilitan el diagnóstico presuntivo pre-operatorio, el estudio citológico del material aspirado por punción del Douglas, la culdoscopia y la laparoscopia. La histerosalpingografía puede diseminar las células tumorales a la cavidad y solo serían informadas como salpingitis crónica o hidrosalpinx (2). Ingram, recomienda como lo más efectivo, el examen pélvico de rutina mínimo cada $6 \mathrm{me}$ ses (13).

Tabla No. 4

DIAGNOSTICO PRESUNCIONAL ANTES DE LA INTERVENCION

\section{SEGUN VARIOS AUTORES}

\begin{tabular}{|l|r|rc|}
\hline \multirow{2}{*}{ Autores } & \multirow{2}{*}{$\begin{array}{c}\text { No. } \\
\text { Total }\end{array}$} & \multicolumn{2}{|c|}{$\begin{array}{c}\text { Diagnóstico } \\
\text { presuntivo }\end{array}$} \\
\cline { 3 - 4 } & & No. & $\%$ \\
\hline Turunem & 58 & 3 & 5.2 \\
Urrutia & 6 & 2 & 33.3 \\
Muntazze & 8 & 1 & 12.5 \\
Jones & 780 & 10 & 1.3 \\
Andrade & 3 & 0 & 0 \\
Hershey & 16 & 5 & 31.2 \\
\hline Total & 871 & 21 & $2.41 \%$ \\
\hline
\end{tabular}


Un estudio reciente del Malmo General Hospital de la universidad de Lund en Suecia, informa como signo importante en el diagnóstico temprano del carcinoma de trompa, la presencia de productos de degradación del fibrinógeno (FDP) elevados, en el material de legrado uterino y en el flujo de descarga vaginal acuosa. También se ha informado FDP elevado en casos de tumores malignos del ovario por Suanberg L. y Asted $B$. en el líquido ascítico y en el material de citología y flujo vaginal y por Niklasson, en casos de adenocarcinoma endometrial (20).

Los síntomas que más comunmente han sido descritos por los diferentes autores en orden de frecuencia son: 1. dolor pélvico; 2 . descarga vaginal acuosa; 3. masa pélvica; 4. sangrado anormal. (Tabla No. 5).

En la clasificación clínica tomaremos los estados clínicos recomendados por Dodson y colaboradores y que con similares a la clasificación de la FIGO para tumores ováricos (18) (21), aún cuando hay autores que consideran in situ o estado cero, el limitado a la mucosa (11).

Tabla No. 5

\section{SINTOMAS MAS FRECUENTES}

\begin{tabular}{|l|l|}
\hline \multicolumn{1}{|c|}{ Autor } & \multicolumn{1}{c|}{ Síntomas } \\
\hline Andrade & $\begin{array}{l}\text { Dolor - Descarga vaginal acuosa y masa pélvica. } \\
\text { Hemorragia anormal. Dolor pélvico y masa pélvi- } \\
\text { ca. } \\
\text { Hemorragia anormal. Leucorrea acuosa y dolor } \\
\text { pélvico. }\end{array}$ \\
Ingram & $\begin{array}{l}\text { Descarga vaginal acuosa y dolor pélvico. } \\
\text { Sangrado anormal. Descarga vaginal acuosa. } \\
\text { Tamimi }\end{array}$ \\
Anderson pélvico y masa pélvica. \\
Hershey & $\begin{array}{l}\text { Dolor pélvico y masa pélvica. } \\
\text { Dolor pélvico. Sangrado anormal y leucorrea } \\
\text { Molor pélvico. Descarga vaginal acuosa y masa }\end{array}$ \\
\hline
\end{tabular}

\section{Estado I:}

A.Crecimiento limitado a una trompa sin ascitis.

B. Crecimiento limitado a las dos trompas sin ascitis.

C. Crecimiento en una o en las dos trompas, ascitis presente con células malignas en el líquido.

Estado II: Crecimiento que afecta a una o ambas trompas con extensión pélvica.

A. Al útero o a los ovarios.

B. A otros tejidos pélvicos.

Estado III: Crecimiento en una o ambas trompas, con metástasis intraperitoneales difusas al abdómen.

Estado IV: Crecimiento a una o ambas trompas, con metástasis a distancia fuera de la cavidad peritoneal.

\section{ANATOMIA PATOLOGICA}

En el $96 \%$ de los casos los tumores malignos de las trompas de falopio son adenocarcinomas (23).

Macroscópicamente la trompa se encuentra aumentada de tamaño a veces en grado extraordinario. En este último caso puede parecer una piosalpinx enorme, que por lo general se halla completamente libre de adherencias con los órganos vecinos, a diferencia de lo que ocurre en la enfermedad inflamatoria pélvica (EIP). La trompa contralateral suele ser normal, exceptuando los casos raros de tumor bilateral, a diferencia también de la EIP, en la cual el proceso es casi siempre bilateral (21).

Microscópicamente la imágen típica corresponde a una formación papilar que se insinúa concéntricamente en la luz del órgano, que presenta muy poca o ninguna tendencia a invadir la serosa a 
través de la túnica muscular. Una variedad menos común es la alveolar en la cual la fusión de los pliegues papilares simulan una estructura glandular, (6), (21). Hay autores como Ryan, Paverstein y Woodruff, que sugieren la existencia de carcinomas realmente iniciales in situ de la trompa y otros que la coexistencia de carcinoma in situ del cervix con carcinoma in situ de la trompa, enfatiza la noción de neoplasias "multicéntricas" en las diferentes estructuras de origen Mülleriano (19). El epitelio del tumor es secretor, con células ciliadas intercaladas con no ciliadas con granulos de secreción en el citoplasma (14). La ultraestructura de los adenocarcinomas de trompas muestra células con abundantes mitocondrias, con numerosas microvellosidades hacia la luz, pero exentas de verdaderas cilias y conteniendo material de secreción (25). El microscopio electrónico revela diferencias con el adenocarcinoma papilar bien diferenciado; sus células tienen núcleo pleomórfico, múltiples y gigantes nucleólos, numerosas mitocondrias, pobre diferenciación celular, abundantes polisomas y pequeño número de células ricas en lisosomas. EI epitelio normal de la trompa está constituído por células ciliadas, células secretoras y células indiferenciadas escasas. En cambio este tumor no tiene casi células ciliadas, tiene pocas células secretoras y muchas células indiferenciadas, lo que indica su origen en este tipo de células (22).

Su propagación la hace por: 1 - Vía linfática; 2 - Metástasis intracanalicular; 3. - rara vez por continuidad al ovario (21).

Un estudio de Tamimi encontró propagación linfática en el $53 \%$, con propagación a ganglios para-aórticos en $33 \%$, lo cual se considera como dato de mal pronóstico. (29) La vía intracanicular la demuestran los casos de extensión a endometrio, publicados por Volmer (32) y Tjiptohardjo (30). La extensión al ovario la demuestra la publicación de Márquez. (18).

Hay casos de metástasis raras, como por ejemplo, al ombligo, en una paciente de 75 años con hiperplasia endometrial atípica al legrado y masa anexial palpable, en quién la laparotomía reveló adenocarcinoma primario de trompa izquierda, estado IV (10).

Se han descrito tres grados histológicos: grado I: papilar con escasas mitosis; grado II: alvéolo-papilar con formaciones glandulares y grado III: alvéolo medular con masas sólidas (18).

Los criterios para identificar el adenocarcinoma de trompa como primario son: (18).

1. El epitelio del endosalpinx debe ser remplazado en parte o en su totalidad por el adenocarcinoma.

2. El carácter histológico debe ser similar al del epitelio del endosalpinx.

3. El endometrio y los ovarios pueden ser:

\section{a. normales}

b. afectados por proceso maligno diferente.

c. lesión maligna pequeña, que por sus caracter ísticas histológicas parezcan metástasis del tumor de la trompa.

4. Tumor del endosalpinx. El peritoneo tubario, los linfáticos de la capa muscular $y$ el mesosalpinx rara vez están invadidos.

5. La TBC tubárica debe ser excluída ciudadosamente.

\section{PRONOSTICO}

Es dentro de las neoplasias malignas ginecológicas, la menos curable y su pronóstico es sombrío (2). La sobrevivencia en relación al grado histológico, no ha podido ser establecida debido a su extre- 
ma rareza; (18) en cambio la sobrevivencia sí tiene una relación directa con el diagnóstico temprano y su estado clínico. Los estados clínicos I-A y I-B son los de mejor pronóstico (4), (5), (11), (16), siempre y cuando la serosa de la trompa no esté rota, que es considerado por varios autores como un signo ominoso (13), (16). La expectativa de vida en los estados clínicos IV es apenas de dos años con tratamiento adecuado (15).

En nuestra revisión bibliográfica la sobrevivencia a 5 años con tratamiento adecuado, fue en promedio de $37.5 \%$. (Tabla No. 6).

Tabla No. 6

\section{PRONOSTICO}

\begin{tabular}{|l|c|c|}
\hline Autor & $\begin{array}{c}\text { Referencia } \\
\text { Biblio- } \\
\text { gráfica }\end{array}$ & $\begin{array}{c}\text { Porcentaje } \\
\text { de } \\
\text { sobrevivencia } \\
\text { a 5 años }\end{array}$ \\
\hline Engstre & 2 & $38 \%$ \\
Ross & 2 & $40 \%$ \\
Hayden y Polter & 2 & $27 \%$ \\
Engeler & 8 & $30 \%$ \\
Hershey & 11 & $44 \%$ \\
Kubista & 17 & $26 \%$ \\
Kneale & 21 & $40 \%$ \\
Hanton & 21 & $45 \%$ \\
Pérez & 23 & $40 \%$ \\
Raju & 24 & $48 \%$ \\
Sedlis & 26 & $34 \%$ \\
\hline
\end{tabular}

$\mathrm{X}: 37.5 \%$

\section{TRATAMIENTO}

El tratamiento más recomendado es la histerectomía abdominal total con salpingooforectomía bilateral más radioterapia o quimioterapia post-operatorias, aunque para algunos autores su valor sea dudoso (17), (21). Wolf recomienda. agregar al tratamiento quirúrgico, oro radio-activo intra-peritoneal que, según él, mejora la sobrevivencia a 5 años (33).

En los estados clínicos iniciales (estado 0 y estado 1), parece no existir diferencia significativa entre el tratamiento quirúrgico solo, 0 agregado de radioterapia (2), (11), (17).

En casos avanzados las opiniones están divididas, entre si se agrega a la cirugía quimioterapia (2), (7), o radioterapia (6), (23), o es indiferente el que se agregue quimioterapia o radioterapia (11), (18).

\section{Presentación del caso}

Paciente de 47 años, grávida 5:, partos 4, abortos 1. Menopausia hace 2 años. Antecedente de colecistectomía hace 18 años. Consulta en enero 21 de 1982 , por sensación de masa abdominal desde hace 4 meses y flujo amarillo, en ocasiones achocolatado, de 2 años de evolución. En los últimos 2 años, sangrado genital intermitente escaso.

Al examen de abdómen se encuentra cicatriz de colecistectomía y gran masa abdominal irregular y nodular que ocupa toda la pelvis, flancos $y$ asciende hasta epigastrio; su volumen aproximado es de $25 \mathrm{~cm}$ de diámetro. Genitales externos normales. Especulos'copia: cuello sano, flujo amarillo. Tacto vaginal: vagina normal, cuello anterior cerrado, fondos del saco y pelvis ocupados por la masa descrita en abdómen.

Laboratorio: Citología pap. II; FV: tricomonas; parcial de orina normal; urocultivo negativo; cuadro hemático, nitrógeno uréico, creatinina y glicemia normales; grupo sanguíneo $0, \mathrm{RH}$ positivo.

Urografia: Ausencia de gas en ampolla rectal, con aumento de la densidad de 
tejidos blandos que sugiere masa en región hipogástrica. El urograma muestra fase nefrográfica retrasada en el lado derecho, con distensión pielo-calicial por pielonefritis. La imágen pielo-calicial izquierda normal; vejiga deprimida en su techo. En la placa en posición vertical, se observa ectasia ureteral derecha.

\section{Electrocardiograma: Normal.}

Tórax: Parénquima pulmonar e imagen cardiovascular radiológicamente normal. No hay adenopatías mediastinales, como tampoco imágen de metástasis.

Con diagnóstico de leiomiomatosis uterına se practica laparatomía, encontrando un gran quiste de ovario derecho de $25 \mathrm{~cm}$ de diámetro de contenido achocolatado, adherido firmemente al recto-sigmoide. En el proceso de liberación la masa se rompe, saliendo material líquido achocolatado muy abundante que se aspira para facilitar la cirugía. También se produce pequeña lesión de $2 \mathrm{~cm}$ en la pared anterior del rectosigmoide, la cual se aisla para cierre posterior a la ectomía. Las dos trompas uterinas están amorcilladas la derecha de $20 \mathrm{~cm}$ de longitud cabalgando y firmemente adherida al quiste de ovario derecho. La trompa izquierda mide 12 $\mathrm{cm}$ de longitud, adherida a la pared posterior del útero; ovario izquierdo atrófico.

Utero aumentado dos veces su tamaño $y$ deformado por dos leiomiomas subserosas. Se practica histerectomía total con salpingooforectomía bilateral y terminada la ectomía, se repara la lesión del recto-sigmoide la cual queda retroperitoneal, dejándose tubo de drenaje retroperitoneal que se saca a vagina a través del cierre en corona de la cúpula.

\section{ANATOMIA PATOLOGICA}

\section{Descripción macroscópica}

Anexo derecho con masa quística colapsada de $14 \times 14 \mathrm{~cm}$, rota en un extremo, superficie externa gris rojizo irregular con bandas de adherencias, la superficie interna es lisa, en algunos sitios granulosa de color parduzco. Firmemente adherida a esta masa se encuentra formación piriforme de $13 \mathrm{~cm}$ de longitud y $6 \mathrm{~cm}$ de diámetro, en su parte distal más ensanchada y que se comunica directamente con la masa quística, su superficie externa lisa de color gris azulado que al corte se encuentra llena de material friable con extensa necrosis de color parduzco amarillento con áreas de tejido blanquecinogrisáceo.

Trompa izquierda de $10 \mathrm{~cm}$ de longitud y $3 \mathrm{~cm}$ de diámetro, con adherencias en su superficie externa, pabellón sellado e invertido, al abrir la trompa se encuentra material hemorrágico y fragmentos sueltos de tejido friable grisáceo blanquecino; superficie interna con nodulaciones irregulares de tejido blanquecino friable, ovario izquierdo de $3 \times 7$ con adherencias externas $y$ al corte parénquima elástico grisáceo.

Utero de $112 \mathrm{gr}$. de peso $\mathrm{y}$ de $11 \times 5 \times 2.5 \mathrm{~cm}$. En la superficie serosa 2 leiomiomas de 2 y $2.5 \mathrm{~cm}$. de diámetro; cervix de $4 \times 2 \times 2.5$, con exocervix liso y orificio externo rasgado de $1 \mathrm{~cm}$; canal endocervical sin cambios; cavidad uterina de $6 \times 5 \mathrm{~cm}$, escaso endometrio de aspecto uniforme liso rojizo y de 0.2 a $0.3 \mathrm{~cm}$; miometrio de $1.3 \mathrm{~cm}$, con leiomioma intramural de $1 \mathrm{~cm}$ de diámetro.

\section{Descripción microscópica}

En el gran quiste colapsado se identifica parénquima ovárico, con focos de inflama ción crónica en la pared. En la luz, fragmentos sueltos de adenocarcinoma medianamente diferenciado; ovario izquierdo con atrofia y tejido fibroso adherido a su superficie. Cervix uterino con inflamación crónica no específica. Los cortes de endometrio muestran endometroide de 
tipo proliferativo y leiomiomas intramurales. No hay malignidad en el endometrio.

Los cortes de trompas uterinas muestran pared adelgazada y mucosa distendida y aplanada; en áreas la mucosa está remplazada por adenocarcinoma medianamente diferenciado, sólido, con tendencia papilar focal. El tumor crece hacia la luz e invade en áreas superficialmente la pared; el tumor muestra extensas zonas de necrosis.

En vista de las características macro y microscópicas del tumor, se clasifica como primitivo bilateral de las trompas uterinas ya que no se encontró tumor en el útero u ovarios; el quiste ovárico derecho no se puede clasificar bien, pero en él no se observó adenocarcinoma presente en su superficie interna, por lo cual se cree que se trata de un quiste endometriósico antiguo.

\section{DIAGNOSTICO}

1. Adenocarcinoma primario bilateral de trompas uterinas, medianamente diferenciado tipo papilar.

2. Quiste endometriósico antiguo de ovario derecho.

3. Adenomiosis

4. Leiomiomatosis

\section{EVOLUCION}

La paciente recibió en el post-operatorio inmediato, tratamiento antibiótico para cubrir gram positivos, gram negativos $y$ anaerobios. Su evolución fue satisfactoria y al 6o. día de postoperatorio se retiró el tubo vaginal de drenaje. El 7o. día se autorizó salida recibiendo a partir del día 15, tratamiento con telecobaltoterapia hasta completar 6.000 rads. A los 6 meses de la intervención, la paciente se encuentra bien y libre de tumor.

\section{RESUMEN}

Se presenta un caso de adenocarcinoma primario bilateral de trompas uterinas, tumor extremadamente raro cuya frecuencia oscila del $0.1 \%$ al $1.4 \%$ de todas las neoplasias ginecológicas malignas. Su tratamiento fue histerectomía total más salpingo-oforectomía bilateral seguida de radioterapia.

Se hace una extensa revisión bibliográfica mundial analizando la historia del tumor, su frecuencia, la edad $y$ paridad de las pacientes que la sufren, el diagnóstico y sintomatología, la anatomía patológica y su pronóstico $y$ tratamiento. Se presenta además su clasificación clínica e histológica aceptada internacionalmente por la mayoría de los autores.

\section{SUMMARY}

It is shown a case of a primary bilateral adenocarcinoma of the uterine tubes, a tumour too much rare which frecuency oscillates from $0.1 \%$ to $1.4 \%$ of the whole malignant neoplasias. Its treatment was total hysterotomy plus bilateral salpingooforectomía follows by radiotherapy.

After six months of treatment, the patient is in good condition and free of tumour.

It is done a wide world bibliographic revision, analising the tumour history, its frecuency, the age and parity of the patients who suffer it, the diagnostic and symptomatology, the pathologic; anatomy and treatment. Besides, it is shown its clinical and histological classification which has been accepted internationally, by most of the authors.

\section{BIBLIOGRAFIA}

1. ANDERSON K.A.; TANAGHO E.A. "Fallopian tube carcinoma associated with retroperitoneal fibrosis". J. Urol. 1981 Jan; 125 (1): 119-21 
2. ANDRADE A.; CASASOLA J.; NAHAMAD M.; MORENO H.; ALONSO G. "Carcinoma primario de la trompa de falopio. Presentación de tres casos clínicos". Ginec. Obstet. Mex. Abril 1979 Vol. 45 año XXXIV (270): 299-309.

3. BARKHATOVA T.P. "Cancer of the fallopian tubes". Feldsher Akush 1981; 46 (9): 55-7.

4. BARTL W.; FEICHTINGER W.; BREITENECKER G. "Clinical and Pathological aspect of primary carcinoma of the fallo. pian tube". Wien Klin Wochenschr 1980 May 9; 92 (10): 360-4.

5. BENEDET J.L.; WHITE G.W.; FAIREY R.N.; BOYES D.A. "Adenocarcinoma of the fallopian tube Experience with 41 patients".

6. CANTILLO J.; ROJAS Laura.; MILANES L. "Carcinoma primario de la trompa de falopio. Reporte de dos casos".

7. DEPPE G.; BRUCKNER H.W.; COHEN C.J. "Combination chemoterapy for ad. vanced carcinoma of the fallopian tube". Obst. Gynecol 1980 Oct.; 56 (4): 530-2.

8. ENGELER V.; REINISH E.; SCHREINER W.E. "Primary carcinoma of the fallopian tube a clinical study of $\mathbf{3 7}$ cases". Geburtshilfe Fraunheilkd 1981 May; 41 (5): 325-9.

9. FOX C.H. "Adnexal malignancy detected by cervical citology". AM. J. Obstet. Gynecol 197815 Sep.; 132 (2): 148-50.

10. GALLE P.C.; DOBSON V.W.; HOMESLEY H.D. "Umbilical metastasis from gynecologic malignancies: A primary carcinoma of the fallopian tube". Obstet. Gynecol. April 1981; 57 (4): 531-3.

11. HERSHEY D.W.; FENNELL R.H.; MA. JOR F.J. "Primary carcinoma of the fallopian tube". Obstet. and Gynecol. March 1981·Vol. 57 (3): 367-70.
12. H'OPFEL.KREINER; MIKUS G. "Accidental cytological findings in routine vaginal smear in primary carcinoma of the fallopian tube". Pathol res pract. 1978 Oct.; 163 (2): 163-7.

13. INGRAM F.H.; HISLEY J.C. "Primary carcinoma of the fallopian tube". South Med. J. Sept. 75; 68 (9): 1153-5.

14. JOHNSON L; DIAMOND I; JULLY G. "Ultrastructure of fallopian tube carci. noma". Cancer 1978 Sept; 42 (3): 1291.7.

15. KADZIORA M.B.; SRINIVASAN R. "Primary carcinoma of the fallopian tube". CAN J. Surg. 1981 Jul.; 24 (4): 425-6.

16. KINZEL G.E. "Primary carcinoma of the fallopian tube". AM. J. Obstet. Gynecol. 15 Jul. $76 ; 125$ (6): 816-20.

17. KUBISTA E.; KUPKA S. "Clinical pro. blems, therapy and prophylaxis of primary carcinoma of the fallopian tube". Gebuntshille Frauenheilkd 1977 Dec. 37 (12): 1044.9.

18. MARQUEZ M.A.; PANIAGUA A.; VI. LLALOBOS F.; FERNANDEZ J. "Car. cinoma primario de la trompa de falopio". Ginec-obstet. Mex. Vol. 45 año XXXIV (270): 311.17 abril 1979.

19. MENDEZ J.A.; BEDOYA J.M.; MANTILLA A.; NOGALES F. Jr.; GALERA $H$. "Carcinoma in situ of the fallopian tube associated with cervical carcinoma. 'Case report". INT. J. Gynecol Obstet. 1976; 14 (4): $352 \cdot 5$.

20. NIKLASSON O.; HENRIKSSON L.; STORMBY N.; ULMSTEN U. "Early diagnostic signs in the development of a fallopian tube carcinoma". Acta Obstet. Gynecol. Scand 1980; 59 (1): 93-4.

21. NOVAK. "Tratado de Ginecología". Novena edición. Enero 1977. pág. 423-5. 
22. OKUDA $Y_{\text {,; }}$ OKAMURA H.; KANZAKI H.; KAWAGUCHI K.; SUSUKI A.; NIS. HIMURA T. "An intrastructural study of primary carcinoma of the fallopian tube". Acta Obstet-gynaecol Jpn 1980 Dec.; 32 (12): 1927-36.

23. PEREZ S.M. "Detección precoz diagnós. tico y tratamiento del cáncer". Madrid 1965. pág. 290-2.

24. RAJU K.S.; BARKER G.H.; WILTSHAW E. "Primary carcinoma of the fallopian tube. Report of 22 cases"., BR. J. Obstet. Gynaecol 1981 Nov.; 88 (11): 1124-9.

25. RORAT E.; FENOGLIO C. "The ultras. tructure of a poorly differentiated adeno. carcinoma of the human tuba uterina". Oncology 1976; 33 (4): 167-9.

26. SEDLIS A. "Carcinoma of the fallopian tube". Surg. Clin North AM 1978 Feb.; 58 (1): 1219

27. SHINFELD J.S.; WINSTON H.G. "Prima. ry tubal carcinoma in pregnancy". AM J. Obstet. Gynecol 1980 Jun. 15; 137 (4): 512.4 .

28. STARR A.J.; RUFFOLO E.H.; VITTAL B.; MARSTON B.R. "Primary carcinoma of the fallopian tube: A suprise finding in a postpartum tubal ligation". AM. J. Obstet. Gynecol October 1, 1978. Vol. 132 (3): 344.5.

29. TAMIMI H.K.; FIGGE D.C. "Adenocar. cinoma of the uterine tube: potential for lymph node metastases"..".

30. TIJPTOHARDJO T.A.; GISSER S.D. "Primary adenocarcinoma of the fallo. pian tube. A case report with discussion of its pattern of endometrial spread and possible resulting diagnostic confusion". Diagn Gynecol Obstet. 1981 spring; 3 (1): $45 \cdot 8$.

31. VINALL P.S. "Primary carcinoma of the fallopian tube associated with tubercu. lous salpingitis. A case report". BR. J. Obstet. Gynec. 86 (12): 948-9. Dec. 1979.

32. VOLMER J. "Bilateral tube carcinoma with unusual in involment of the endo. metrium". Zentralbl Gynaekol 1975 (21): $1310 \cdot 3$.

33. WOLF A.; VAHRSON H. "Radioactive gold treatment of primary carcinoma of the fallopian tube". Geburtshilfe Frauen. heilkd Feb. 76; 36 (2): 178-83. 